\title{
Aplicación de un modelo hidrológico espacialmente distribuido en dos cuencas costeras de la Región de La Araucanía (Chile $\left.{ }^{1}\right)$
}

\section{Application of a spatially distributed hydrological model in two coastal basins in the coastal region of Araucanía (Chile)}

\author{
Eduardo Fernández-Soto ${ }^{2}$ y Fernando Peña-Cortés ${ }^{3}$
}

\section{INTRODUCCIÓN}

La modelación hidrológica es una herramienta que utiliza ecuaciones matemáticas para representar, en diverso grado de detalle, las complejas interacciones del agua, la energía, el relieve y la vegetación, generando una simplificación del funcionamiento del sistema natural (Viviroli et al., 2009). Al respecto, son dos los tipos de modelos más frecuentemente utilizados: los modelos de tipos analógicos o conceptuales y los modelos físicos. Éstos últi-

\footnotetext{
${ }^{1}$ Los autores agradecen a Jimmy Pincheira por el apoyo en la edición y revisión crítica del manuscrito y el financiamiento del proyecto FONDECYT 1151375.

2 Eduardo Fernández-Soto es Magíster en Planificación y Gestión Territorial por la Universidad Católica de Temuco (Chile). Investigador del Laboratorio de Planificación Territorial, Facultad de Recursos Naturales, Universidad Católica de Temuco (Chile), Casilla 15-D. efernandez@uct.cl +56-45-2205469.

${ }^{3}$ Fernando Peña-Cortés es Doctor en Ciencias Ambientales por la Universidad de Concepción (Chile). Investigador del Laboratorio de Planificación Territorial y Núcleo de Investigación en Estudios Ambientales, Facultad de Recursos Naturales, Universidad Católica de Temuco (Chile), Casilla 15-D. fpena@uct.cl
} 
mos pueden clasificarse en determinísticos, cuando se conoce el resultado en forma puntual sin incertidumbre, y en estocásticos, cuando son probabilísticos. Asimismo de acuerdo al origen de la información para su construcción se dividen en modelos heurísticos, basados en las explicaciones sobres las causas de un fenómeno, y en modelos empíricos, que se basan en observaciones directas experimentales de los fenómenos (Himmelblau y Bischoff, 2004). Dentro de los modelos matemáticos físico-estocásticos se reconocen dos grandes grupos: Los modelos agregados, que tratan de obtener una estimación de un set de datos que no puede ser medido directamente, y los modelos espacialmente distribuidos, dónde se da un valor a cada unidad espacial (e.g., cuencas hidrográficas) en función de criterios hidrológicos.

La necesidad de modelar el comportamiento hidrológico cobró relevancia con la hidrología estocástica o estadística desde la década de los 70's. Esta aproximación se fundamenta en la recopilación de las variaciones aleatorias que presentan los fenómenos hidrológicos en el tiempo, para estimar la influencia que estas variaciones tienen en los procesos hidráulicos que impactan sobre el territorio, la economía y la sociedad (Campos, 1998; Pedregal, 2005). Esta aproximación considera que los eventos hidrológicos ocurridos en el pasado, y los que tendrán lugar en el futuro, son funciones muestrales de procesos estocásticos muy complejos, expresados como función aleatoria de un factor que generalmente es el tiempo (Moreno y Salazar, 2009).

Inicialmente estos modelos estocásticos sólo podían aplicarse puntualmente en un espacio geográfico reducido, sin embargo, con las nuevas potencialidades de los Sistemas de Información Geográfica (SIG), la modelación ocurre de forma más rápida y automática casi en cualquier escala y de forma distribuida en el espacio (Boyko y Treebushny, 2006). Estos modelos, necesitan fuentes de información espacial, las cuales son cada vez más masivas, gracias a los avances técnicos que se han desarrollado, el mejoramiento constante de las interacciones de los SIG con los modelos hidrológicos matemáticos, la disponibilidad de datos espaciales, y la disminución de los costos para el levantamiento de información territorial (Llorente et al., 2009).

En este sentido, la integración SIG-Modelación hidrológica permite hacer una caracterización morfométrica de las cuencas. Para ello se utilizan como base de datos los Modelos de Elevación Digital (DEM) que constituyen una representación de la topografía del terreno (Ávila, 2002), a distintas resoluciones espaciales, y que permite por ejemplo la delimitación de cuencas, la construcción de las redes de drenaje y la identificación de la dirección de escurrimientos (Müller-Wohlfeil et al., 1996). Además, las herramientas de modelamiento espacial en los SIG (e.g., ArcHydro Tools) permiten estimar y 
generar la distribución espacial de parámetros hidrológicos a partir de la aplicación de diferentes técnicas geoestadísticas como la interpolación (e.g., Kriging, Spline, IDW) (Mendoza et al., 2002).

La calidad de las simulaciones hidrológicas, depende de la capacidad del modelo de base para describir y representar con exactitud la heterogeneidad de estos sistemas hidrológicos en las diferentes escalas espaciales y temporales (Viviroli et al., 2009). Así, un modelo puede simular los flujos medios mensuales en régimen natural de cualquier punto en una cuenca o área de estudio, siendo utilizada para una serie de procesos, como la predicción de inundaciones (Llorente et al., 2009; Rovira y Latorre, 1986; García et al., 2002; Al-sabhan et al., 2003; Olivares, 2004), la modelación hídrica en áreas montañosas (Moussa et al., 2007), la evaluación del recurso hídrico superficial y subterráneo (Sharma y Anjaneyulu, 1993; Thunnissen et al., 1992; Allewijn, 1988; Llorens et al., 2003; Hesse et al., 2008), la predicción de la pérdida de suelos y erosión (Sharma y Singh, 1995; Choi y Deal, 2008), la reconstrucción de comportamiento en paleohidrología (Conde y Baena, 2004), el manejo de incertidumbre hidrológica para la operación de sistemas energéticos (Palacios, 2004), la reconstrucción de series de datos (Moreno y Salazar, 2008; Moreno y Salazar, 2009) y la ordenación del territorio (Maza et al., 1995) entre otras aplicaciones.

Estas aplicaciones dependen de la disponibilidad de datos de las estaciones de aforo, de la calidad de la información meteorológica y las características de las cuencas y acuíferos (Loaiza, 2007; Pizarro et al., 2005). No obstante, cuando no se cuenta con esta información o existen antecedentes parciales del comportamiento de una cuenca se hace muy compleja su simulación (Belmonte y Núñez, 2006). Cuando esto ocurre se puede integrar información multitemporal de datos hidráulicos, meteorológicos, además de datos topográficos, geológicos, y tipos y usos de suelo existentes (Núñez et al., 1998). Lo anterior permitiría prescindir en cierta medida de aforos y series de datos complejos que encarecen la modelación, particularmente en zonas de poco interés económico.

\section{ProblemÁticA}

La inexistencia de datos es muy común en países subdesarrollados, principalmente porque la instalación, mantención y manejo de los datos es un proceso costoso (Mendoza et al., 2002). En el borde costero de la región de La Araucanía en Chile, existen pocos aforos que permitan obtener información de las características de los cursos de agua existentes, como el caudal y la ve- 
locidad de flujo, entre otros. Sólo se encuentran presentes las estaciones de la DGA (Dirección General de Aguas) perteneciente al MOP (Ministerio de Obras Públicas), las cuales resultan insuficientes para comprender la dinámica hídrica de las cuencas costeras locales. Es por ello que surge la necesidad de levantar la información mediante métodos indirectos, como ocurre con la modelación hidrológica espacialmente distribuida (Mendoza et al., 2002) y su integración en plataformas SIG.

Este tipo de modelación toma un especial interés para la planificación del uso del recurso hídrico en una zona agrícola y forestal que presenta elementos biofísicos y socioculturales particulares, como la alta presencia de humedales (Peña-Cortés et al., 2006; Peña-Cortés, Escalona, Pincheira-Ulbrich y Rebolledo, 2011; Peña Cortés, Pincheira-Ulbrich, Bertrán, et al., 2011), alta proporción de población mapuche (>50\%) (INE, 2002), elevados índices de pobreza y matrices productivas de subsistencia altamente degradadas (MIDEPLAN, 2006, Peña-Cortés et al., 2009, CASEN, 2009). Así en este estudio se desarrolla una modelación espacialmente distribuida para la cuenca del río Moncul y lago Budi en la Región de La Araucanía, dos regiones que presentarían dinámicas diferenciadas dadas por su morfogénesis (Peña-Cortés et al., 2014). El estudio define los flujos hídricos, la red de drenaje y la regionalización de las subcuencas hidrográficas. Adicionalmente, para comprender la dinámica de ambas cuencas se caracterizó la cobertura del suelo, el tamaño y forma de las cuencas, pendiente, altitud y forma red hídrica.

\section{ÁREA DE ESTUDio}

El área de estudio corresponde a las cuencas del Río Moncul y del lago Budi, localizadas en el borde costero de la región de La Araucanía en Chile, ambas de alimentación pluvial. La cuenca del río Moncul se ubica al norte, en la comuna de Carahue, posee una superficie de 45.622 ha y ocupa el 34\% de la superficie comunal. Desemboca en el río Imperial al oeste de la localidad de Nehuentúe y su cabecera se ubica en el margen sur del cordón montañoso de la Cordillera de Nahuelbuta.

La cuenca del Lago Budi se localiza en el centro del área de estudio, principalmente en la comuna de Saavedra, con una superficie de 49.083 ha, nace en el relieve de cordones y plataformas de erosión y desemboca hacia el océano Pacífico (figura 1).

En la zona predomina el clima mediterráneo perhúmedo, con precipitaciones medias anuales aproximadas de 1200 mm (Di Castri y Hayek, 1975). 
FIGURA 1

ÁREA DE ESTUDIO

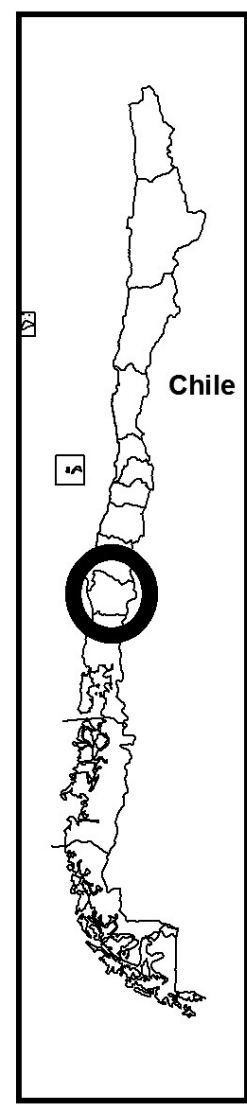

\begin{tabular}{cc}
$73^{\circ} 25^{\prime} 30^{\prime \prime} \mathrm{O}$ & $73^{\circ} 15^{\prime} 0^{\prime \prime O}$ \\
\hline
\end{tabular}
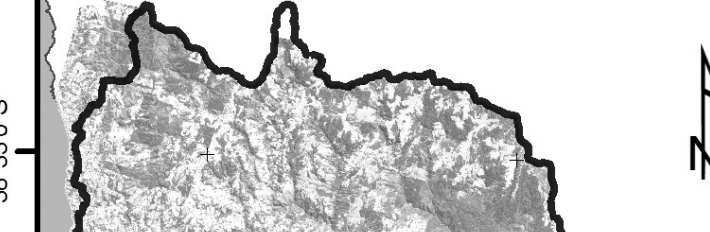

$N$

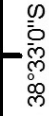

Moncul

45 Región de La Araucanía
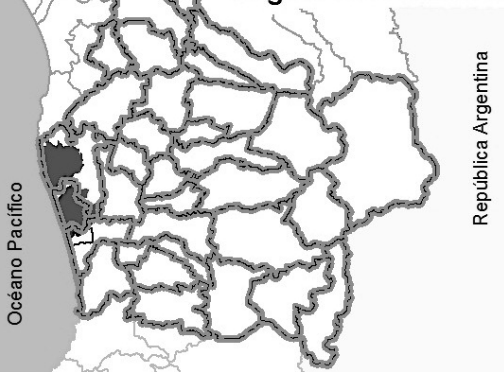

(1)

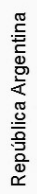

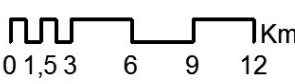

01,53 
MATERIAL Y MÉTODOS

\section{Información de base y variables del modelo}

Las bases cartográficas utilizadas fueron IGM 1:50000 cobertura regular, digitalizadas y reproyectadas en el sistema de coordenadas universal transversal de Mercator (UTM), Datum y elipsoide de referencia WGS-84. Estas fueron utilizadas con los parámetros de transformación del IGM mediante el software CPR\&SIG (Centro de estudios de Percepción Remota y SIG) (Pattillo y Pezoa, 1998). Se utilizó el DEM del sensor ASTER (Advanced Spaceborne Thermal Emission and Reflection Radiometer) de resolución 30m x 30m de píxel referenciado en Datum WGS 84, con una estructura de 16 bits. La determinación de los cuerpos de agua se determinó mediante el proceso de clasificación supervisada de imágenes satelitales (Chuvieco, 2008).

\section{Antecedentes metodológicos}

La modelación hidrológica se elaboró mediante una simulación espacialmente distribuida, que generó un modelo de datos hídricos. Esto permitió la regionalización de unidades de cuenca y subcuenca vertiente (Strahler, 1989) y la definición del sistema de drenaje de la cuenca (Maidment, 2002). Los datos de entrada (DEM-ASTER, red hídrica y cuerpos de agua) y la ejecución del modelo se llevó a cabo mediante la extensión ArcHydro Tools 9 sobre el programa ArcGIS 9.3.1, siendo complementado con el módulo WARPHydro Tools para mejorar la precisión de la regionalización (figura 2). Estas extensiones poseen una estructura estándar para almacenar información hidrológica y meteorológica espacial y numérica en una geodatabase (Gopalan, 2003; Maidment, 2002). La herramienta permite sintetizar la información espacial y temporal de recursos hídricos para soportar análisis y modelamiento hidrológico.

\section{Modelo hidrológico}

El proceso de modelación hidrológica se realizó de acuerdo al siguiente flujo metodológico: i) primero se preparó la información ajustando las coberturas base en proyección y geometría en el Datum WGS-84, y luego se aplicó el método de acondicionamiento del DEM-ASTER (Digital Elevation 
Model - Avanced Spaceborne Thermal Emission and Reflection Radiometer) utilizando la red hídrica y los cuerpos de agua de base. Posteriormente, se corrigió la malla DEM, que consiste en llenar aquellos espacios de error de sumideros (valores muy bajos) y puntas (valores muy altos) para no alterar los resultados de la modelación y eliminar las discontinuidades en la red de drenaje. Esto se realizó con el método de llenado de espacios (Fill sinks). Al momento del acondicionamiento del DEM se probaron dos métodos, el "AgreeDEM" (tradicional) y el "Level DEM". El primero considera el ajuste con la red hídrica de base, mientras que el segundo incorpora además los cuerpos de agua, confiriendo a este último mayor precisión, dada la representatividad que tienen estos cuerpos en las cuencas en estudio. ii) Para el buffer del flujo hídrico se consideraron tres celdas; para las unidades de altitud de caída/subida suaves se consideraron cinco metros (valor de la celda), mientras que para las unidades de altitud de caída/subida fuertes este valor fue de 10 metros (valor de la celda). El comportamiento del ajuste del DEM se visualizó con el programa AgreeMethod v 1.1. El sentido del flujo se obtuvo con el método del modelo D-8 (Deterministic 8) (O Callaghan y Mark, 1984). Este método asume que el agua de un píxel dado tiende a fluir al píxel contiguo a lo largo de la dirección de máxima pendiente, de este modo define la red de dirección de flujo. Posteriormente, para configurar la red de drenaje, se consideraron dos etapas. Primero se calculó la acumulación de flujo, la cuál consideró un valor umbral de tres píxeles. Este umbral fue establecido de acuerdo al enfoque aplicado por Mark (1983). Luego se determinó la red hídrica, en función de la relación entre el área aportante y la pendiente de cada píxel de acuerdo a Tarboton et al., (1991). Esto permitió definir los flujos hídricos o red de drenaje (stream) levantadas a partir del flujo acumulado.

Finalmente, para llevar a cabo el proceso de regionalización se utilizó la extensión WARPHydro para ArcGis (Gopalan, 2003), dada su mayor potencialidad en la precisión espacial para la delimitación de las cuencas. Éste fue aplicado utilizando las bases generadas con ArcHydro, realizando pre-procesos de delimitación de la divisoria de aguas de la cuenca (figura 2). 
FIGURA 2

\section{FLUJO METODOLÓGICO}

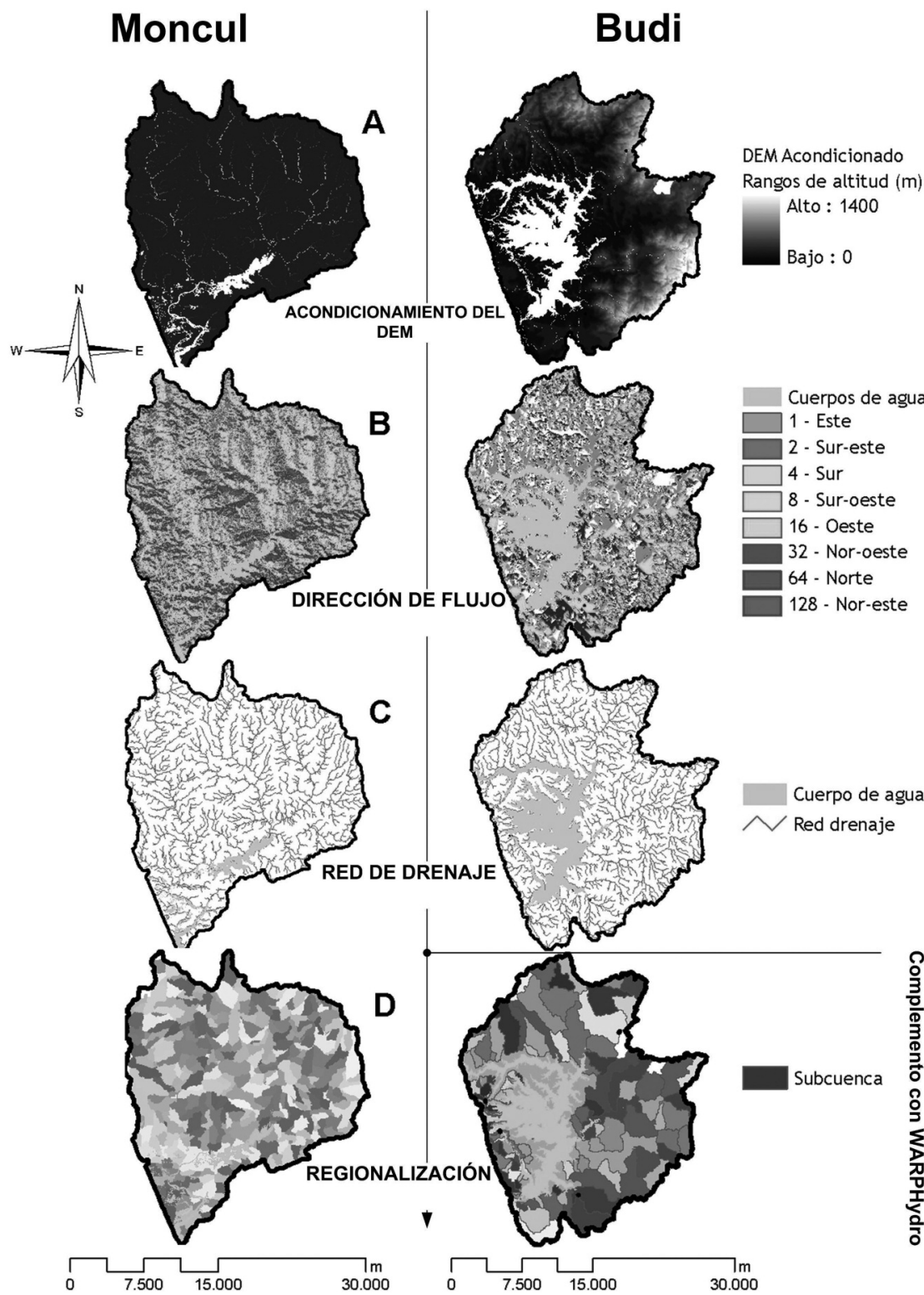

Fuente: Elaborado a partir de Cartografía oficial IGM.

Estudios Geográficos, Vol. LXXVII, 280, pp. 35-56, enero-junio 2016

ISSN: 0014-1496, eISSN: 1988-8546, doi: 10.3989/estgeogr.201602 


\section{Caracterización de las cuencas}

Para comprender la dinámica de las cuencas modeladas se caracterizó el uso de suelo, los parámetros morfométricos, morfológicos e hidrológicos. La información utilizada corresponde al año 2007.

La caracterización de la cobertura de uso de suelo consideró las tres clases de mayor representatividad de superficie en la cuenca, incluyendo las categorías de vegas y de otros terrenos húmedos, ya que son éstas las que mayor volumen de agua almacenan. El uso del suelo se obtuvo mediante geoprocesamiento de las bases cartográficas de cobertura y uso de suelo (CONAF-CONAMA, 2009). Las superficies fueron calculadas con la extensión XTools-Pro v2.0.1 para ArcGis.

Los parámetros morfométricos se evaluaron respecto al tamaño y forma de las cuencas. El tamaño de la cuenca, se clasificó en tres categorías de acuerdo a Jardí (1985): cuenca pequeña (Área $\left.\leq 100 \mathrm{~km}^{2}\right)$, cuenca mediana $\left(100 \mathrm{~km}^{2} \leq\right.$ Área $\leq 2000 \mathrm{~km}^{2}$ ), cuenca grande (Área $>2000 \mathrm{~km}^{2}$ ).

Con respecto a las formas de la cuenca, éstas se evaluaron de acuerdo al índice de Patton (1975), siendo clasificadas en las cinco categorías propuestas por Henao (1988), donde los valores cercanos a uno representan formas redondeadas y los valores cercanas a dos, formas más amorfas (e.g., PincheiraUlbrich et al., 2009) (tabla 1).

TABLA 1

RANGOS DE CLASIFICACIÓN DE ÍNDICE DE PATTON Y SU CLASIFICACIÓN POR HENAO

\begin{tabular}{cc}
\hline Índice Patton & Clasificación de Henao \\
\hline$<1,25$ & Redondo \\
$1,25><1.5$ & Oval redondo oblongo \\
$1,51><1,75$ & Rectangular oblongo \\
$1,76><2$ & Amorfo \\
\hline
\end{tabular}

Fuente: Patton (1975); y Henao (1988). 
En cuanto a los parámetros morfológicos, éstos se caracterizaron respecto de la pendiente y altitud, a partir de la cual se elaboraron perfiles altitudinales de las cuencas mediante una transecta generada con una orientación nortesur, desde la cabecera hasta el cierre. En el caso de la pendiente se aplicaron estadísticas zonales de pendiente media con la extensión Landscape Management System Analyst para ArcGis. Esta caracterización tiene influencia en la velocidad del flujo de agua, que define el tiempo de respuesta de la cuenca.

Para caracterizar la escorrentía superficial, se asumió un comportamiento Hortoniano de las redes modeladas, utilizando el índice de densidad de drenaje, la longitud de flujo superficial (Smart, 1972) y frecuencia de cursos de primer orden, como parámetros hidrológicos. Las mediciones de caudal se realizaron de forma estacional a partir del año 2008 hasta el 2010 con un caudalímetro manual Global Water Flow Probe FP-101.

La clasificación de la forma de la red hídrica se realizó de acuerdo al CEOTMA (1981) que agrupa los flujos en 10 tipos: dendríticos, rectangulares, angulados, pinnados, radiales, anulares, centrípetos, enrejados, paralelos y desordenados.

\section{RESUlTADOS}

\section{Modelo hidrológico}

La modelación espacialmente distribuida definió 84 subcuencas para la cuenca del río Moncul y $1030 \mathrm{~km}$ de flujo hídrico, compuesto por 2350 cauces con características de drenaje dendríticas exorreicas. Para la cuenca del lago Budi se definieron 126 subcuencas y 1147 km de flujo hídrico con características de drenaje centrípeto endo-exorreica (figura 3). Estas diferencias permiten inferir una influencia diferencial en la renovación hídrica entre ambas cuencas. Así, la forma y tipo de drenaje de la cuenca del lago Budi determina un mayor impacto ante un evento de precipitaciones, donde la red hídrica confluye en el lago. En Moncul en cambio, la red confluye hacia el río principal de la cuenca y éste desemboca hacia el río Imperial, se espera por tanto que exista una renovación hídrica más rápida en ésta última cuenca.

De acuerdo al modelo D-8 (O Callaghan y Mark, 1984), los modelos de acumulación obtenidos muestran que la cuenca del Budi tiene una mayor densidad de drenaje lo que implica mayor número de cursos de agua y mayor susceptibilidad a los cambios en la dinámica de la cuenca (figura 3). 


\section{FIGURA 3}

CUENCAS RED HÍDRICA

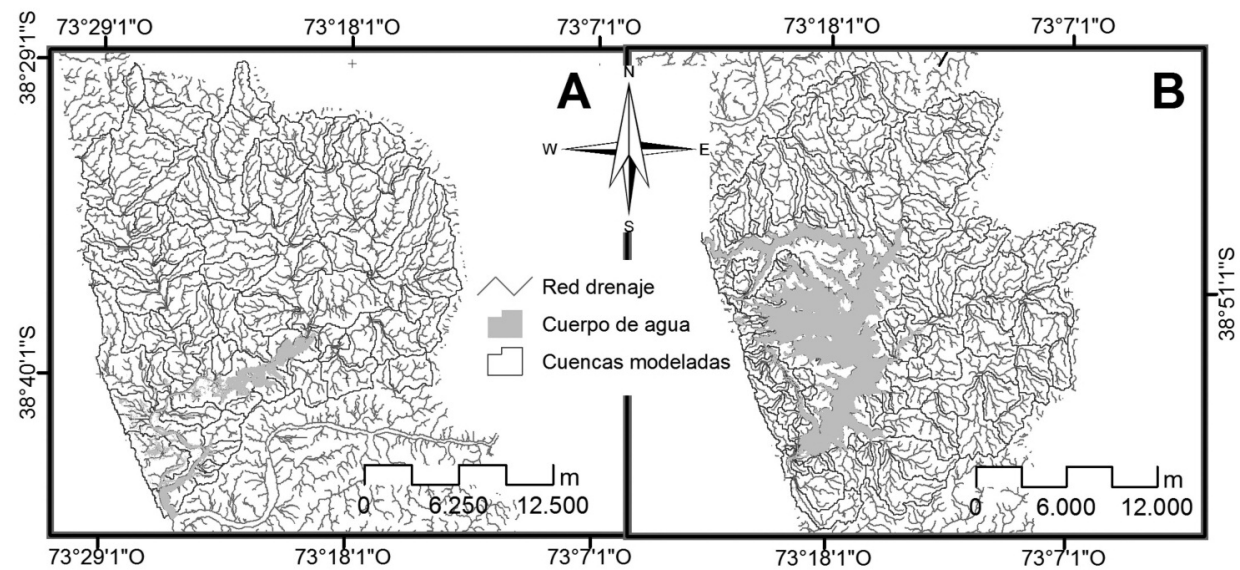

Fuente: Elaborado a partir de Cartografía oficial IGM.

\section{Caracterización de las cuencas}

La cuenca del río Moncul se caracteriza por la presencia plantaciones forestales con un 22\% de ocupación, seguido por la rotación cultivo-pradera con un $15 \%$ y praderas con un 14\%. Aquí los espacios húmedos tienen una representación discreta con un 17\%. En la cuenca del lago Budi en cambio, existe una alta predominancia de la categoría rotación cultivo-pradera con un $66 \%$, seguido de los cuerpos de agua con un 13\%, (debido a la contribución del espejo de agua del lago Budi), y por último la categoría plantación con un 5\%. Estas características pueden tener implicancias importantes en la interceptación y arrastre de material ante eventos de lluvia, debido a la diferencia que tienen ambas cuencas en la estructura de la vegetación (tabla 2).

En relación a los parámetros morfométricos, estos mostraron que en Moncul predominan las cuencas pequeñas con un $89 \%$ de la superficie total, al igual que en la cuenca del Budi con un 62\%. Las formas predominantes de las cuencas fueron las amorfas o fractales, con un índice de Patton de 3, ocupando un 53\% de la superficie total. En la cuenca del Budi se mantiene un patrón similar de formas, donde el $41 \%$ correspondió a las del tipo amorfo (índice 4 de Patton) (tabla 3). Estas características, permiten inferir que ambas cuencas tienen altas posibilidades de presentar grandes caudales en un tiempo reducido después de producido un evento de precipitaciones debido a su simetría. 
TABLA 2

CATEGORIAS DE USO DEL SUELO DE LAS CUENCAS DEL RÍO MONCUL Y RÍO BUDI

\begin{tabular}{|c|c|c|c|c|c|}
\hline \multicolumn{3}{|c|}{ Cuenca del río Moncul } & \multicolumn{3}{|c|}{ Cuenca del río Budi } \\
\hline \multicolumn{6}{|c|}{ Uso del suelo } \\
\hline Categoríal & $\%$ & $\mathrm{Ha}$ & Categoríal & $\%$ & $\mathrm{Ha}$ \\
\hline Plantación forestal & 22,2 & 10.150 & Rotación cultivo-pradera & 66,6 & 32.680 \\
\hline Rotación cultivo-pradera & 15,3 & 7.003 & Cuerpo Agua & 13,3 & 6.542 \\
\hline Praderas & 14 & 6.400 & Plantación & 5,1 & 2.513 \\
\hline Vegas & 5,4 & 2.468 & Vegas & 1,8 & 863 \\
\hline Otros Terrenos húmedos & 12,4 & 5.666 & Otros Terrenos húmedos & 2,9 & 1.433 \\
\hline Otros usos de suelo & 30,5 & 13.933 & Otros usos de suelo & 10,2 & 5.050 \\
\hline Superficie Total cuenca & 100 & 45.622 & Superficie Total cuenca & 100 & 49.083 \\
\hline Superficie Total cuenca & 60,5 & 27,6 & Superficie Total cuenca & 87,4 & 43 \\
\hline
\end{tabular}

Fuente: Uso de suelo, incluye las categorías más representativas de las cuencas caracterizadas utilizando como insumo catastro de CONAF 2007.

TABLA 3

PARAMETROS MORFOMÉTRICOS DE LAS CUENCAS DEL RÍO MONCUL Y RÍO BUDI

\begin{tabular}{|c|c|c|c|c|c|}
\hline \multicolumn{3}{|c|}{ Cuenca del río Moncul } & \multicolumn{3}{|c|}{ Cuenca del río Budi } \\
\hline \multicolumn{6}{|c|}{ Tamaño de las subcuencas ${ }^{1}$} \\
\hline Categoría & $\%$ & $\mathrm{Ha}$ & Categoría & $\%$ & $\mathrm{Ha}$ \\
\hline Pequeñas & 88,9 & 40.587 & Pequeñas & 61,84 & 30354 \\
\hline Medianas & 0,6 & 275 & Medianas & 28,7 & 13828 \\
\hline Grandes & 10,4 & 4.760 & Grandes & 9,9 & 4903 \\
\hline \multicolumn{6}{|c|}{ Forma $^{2}$} \\
\hline Categoría & $\%$ & $\mathrm{Ha}$ & Categoría & $\%$ & $\mathrm{Ha}$ \\
\hline Redondo & 0 & 0,2 & Redondo & 41,4 & 20 \\
\hline Oval redondo & 21,8 & 9.968 & Oval redondo & - & - \\
\hline Oval oblongo & 52,5 & 23.947 & Oval oblongo & 14 & 6872 \\
\hline Rectangular oblongo & 15,7 & 7.155 & Rectangular oblongo & 41,4 & 20315 \\
\hline Amorfo & 10 & 4.552 & Amorfo & 28,5 & 13987 \\
\hline
\end{tabular}

Fuente: Tamaños clasificados de acuerdo a Jardí (1985). Forma índice de Patton (1975) clasificado de acuerdo a Henao (1988). 
Las velocidades de los flujos hídricos variaron entre 0,1 a $1,3 \mathrm{~m} / \mathrm{s}$ en la cuenca del río Budi, y 0,7 a $0,9 \mathrm{~m} / \mathrm{s}$ en la cuenca del río Moncul, lo que implica menor velocidad de arrastre para esta última, por lo tanto, una respuesta más lenta ante un eventual cambio en la dinámica de la cuenca (tabla 3).

Con respecto a las características del perfil altitudinal de las cuencas, se advierte una notable diferencia entre ambas, Moncul presenta un perfil más irregular y con mayor altitud que varía entre $0 \mathrm{~m}$ y $680 \mathrm{~m}$, con una pendiente media de $19,1^{\circ}$. El Budi en cambio, es más homogéneo y con valores de altitud más bajos variando entre 0 y $160 \mathrm{~m}$ (tabla 4), con pendientes que varían un rango más estrecho con una media de $5,8^{\circ}$, esto implica que las respuestas hídricas ocurran con mayor velocidad en Moncul que en Budi ante eventos de precipitaciones.

TABLA 4

PARAMETROS HIDROLÓGICOS DE LAS CUENCAS DEL RÍO MONCUL Y RÍO BUDI

\begin{tabular}{|c|c|c|c|}
\hline \multicolumn{2}{|c|}{ Cuenca del río Moncul } & \multicolumn{2}{|c|}{ Cuenca del río Budi } \\
\hline \multicolumn{4}{|c|}{ Parámetros hidrológicos ${ }^{4}$} \\
\hline Longitud red hídrica $(\mathrm{m})$ & 1.030 & Longitud red hídrica $(\mathrm{m})$ & 1.148 \\
\hline Índice de densidad de drenaje & 2,26 & Índice de densidad de drenaje & 2,34 \\
\hline \multicolumn{4}{|c|}{ Velocidades de los flujos hídricos ${ }^{5}$} \\
\hline Estación & $\mathrm{m} / \mathrm{s}$ & Estación & $\mathrm{m} / \mathrm{s}$ \\
\hline Otoño & 0,7 & Otoño & 1,3 \\
\hline Invierno & 0,8 & Invierno & 0,4 \\
\hline Primavera & 0,9 & Primavera & 0,4 \\
\hline Verano & 0,8 & Verano & 0,1 \\
\hline \multicolumn{4}{|c|}{ Pendiente media } \\
\hline Categoría & Grados & Categoría & Grados \\
\hline Media & $19,1^{\circ}$ & Media & $5,8^{\circ}$ \\
\hline Máxima & $89,9^{\circ}$ & Máxima & $88,7^{\circ}$ \\
\hline Mínimo & $0^{\circ}$ & Mínimo & $0^{\circ}$ \\
\hline Desviación estándar & $27,6^{\circ}$ & Desviación estándar & $13,7^{\circ}$ \\
\hline
\end{tabular}

Fuente: Elaborado de acuerdo a Smart (1972). 
El Budi además de tener respuesta hídrica más lenta, tiene mayor número de cursos de agua, con una densidad de drenaje de 2.34 mientras que Moncul fue de 2.26. Esto lo confirma la frecuencia de cursos de primer orden, donde Moncul presentó 761 cursos, mientras que en el Budi se determinaron 741, esto implica que a mayor cantidad de cursos de orden bajo, son más impetuosos los cursos de agua como respuesta ante un evento (tabla 4).

\section{DisCUSIÓN}

La modelación espacialmente distribuida para la cuenca del río Moncul y lago Budi en la Región de La Araucanía permitió levantar información base para la caracterización de las cuencas, mediante la aplicación de metodologías de evaluación rápida. Este tipo de aproximaciones ha demostrado que la aplicación de modelos espacialmente distribuidos usando el programa ArcHydro, permite una caracterización del comportamiento de las cuencas de manera precisa y a con bajos requerimientos de recursos, tal como lo describe Velásquez et al., (2007). Estos autores utilizaron la capacidad del programa para gestionar datos hidrológicos, además de integrar a éste una interfase de elementos hidráulicos más precisos de modelación, mediciones de caudal, índices de escurrimiento, tiempos de retorno, entre otros, usando para ello los programas HEC-1, HEC-2, HEC-RAS, HEC-HMS, HEC-GeoHMS, HEC-GeoRAS, CRWR-Prepro (Llorente et al., 2009; Núñez et al., 1998).

ArcHydro también se complementa con modelos como el What If? el cual se aplica en la predicción del cambio de uso de suelo y su consecuente cambio hidrológico (McColl \& Agget, 2007). Adicionalmente, ArcHydro se ha utilizado para calibrar modelos como el SWAT, que toman en cuenta las características del suelo, y modelos como el KINEROS que integra tasas de erosión (Pusineri et al., 2005; Miller et al., 2007; Immerzeel y Droogers, 2008) y en la predicción de crecimiento urbano, utilizando estos elementos como base de soporte decisional con programas como HSPF y LEAMluc (Choi y Deal, 2008).

La aplicación del modelo, permitió observar que las diferentes formas y pendientes de las cuencas en ocasiones limitan la precisión del programa para modelar y delimitar las cuencas. Esto ocurre debido a que los algoritmos que utiliza, identifican bien las cotas altas y heterogéneas, pero cuando se trata de cuencas con escasa pendiente este proceso es impreciso y requiere un ajuste asistido, tal como ocurre con la cuenca del Budi. Esta diferenciación ha sido también reconocida por Pusineri et al., (2005) y Olaya (2004). Por su parte, la 
red de drenaje es sensible a las variaciones de escala de trabajo. Cuando se definen escalas grandes $(<1: 10.000)$, la red puede no ser diferenciada, mientras que al definir escalas pequeñas (>1:80.000) ésta se ve muy densa y no se pueden realizar los análisis adecuados, tal como lo describe Ozdemir y Bird (2009). En este estudio parece adecuado trabajar a una escala media de 1:50.000 dada la información base usada y los parámetros ingresados en la modelación.

Las pendientes obtenidas presentaron un comportamiento más homogéneo para el lago Budi con una diferencia entre ellas de 5,5 puntos en su desviación estándar, en relación a lo observado en la cuenca de Moncul, la que tuvo una dispersión de 7,9 respecto a su media. Esto permite inferir que el escurrimiento superficial es mayor para Moncul que para la cuenca del Budi. De acuerdo a ello se observaron características ritrónicas de los cursos de agua, con la marcada presencia del cordón montañoso para la cuenca del río Moncul, en cambio para el caso de la cuenca del Budi las pendientes por las que fluyen los cursos de agua son menores.

Los modelos hidrológicos pueden contribuir en la planificación de los recursos hídricos como insumo base para la toma de decisiones. De acuerdo a ello la National Research Council (NRC 1999) sostiene que para lograr esa contribución y entregar una base científica sólida se requiere la integración de datos, modelos de simulación y la opinión de expertos (Miller et al., 2004).

Con el programa utilizado en esta modelación (ArcHydro), en Estados Unidos y México se han levantado plataformas de información, con modelo de datos que se pueden actualizar, estos datos actualizados logran que los modelos sean más precisos, donde incluso pueden predecir el comportamiento de las cuencas bajo condiciones simuladas, éstas plataformas son utilizadas por los gobiernos locales, quienes tienen una visión global de la dinámica hidrológica para la planificación hidrológica y urbana de las áreas (Velásquez et al., 2007).

La literatura muestra un amplio número de indicadores hidromorfométricos (e.g., índice de sinuosidad, coeficiente Rho, densidad de drenaje, coeficiente de mantenimiento, frecuencia de drenaje, índice de densidad relativa, índice de torrencialidad entre otros, ver Ferrando [1994]), la elección de cualquiera de ellos no sigue una regla formal, sino que está más bien relacionado con las particularidades de las áreas a describir y el objetivo del estudio (Doffo y González Bonorino, 2005), en el presente estudio, los indicadores utilizados, muestran la respuesta hídrica que presentan las cuencas, esto permite inferir algunos componentes de la dinámica de las cuencas, lo que puede complementarse con otras características observadas. 
La demanda de información hidrológica que existe actualmente en Chile es amplia respecto de los recursos hídricos. Sin embargo, la administración de los datos que se levantan no logran tener una sinergia tal que puedan mostrar las dinámicas hidrológicas, en ello radica la importancia de los aforos y la densificación de la red que administre la entidad encarda de ello (en Chile es la Dirección General de Aguas, véase Salazar, 2003). En este estudio se plantea que el modelo hidrológico, como insumo base para la caracterización de la cuenca, es pertinente para el levantamiento de información, siendo posible unirlo tanto a elementos técnicos como en políticas públicas, por ejemplo al Plan de Gestión de Cuencas que se enmarca en la Estrategia Nacional de Gestión Integrada de Cuencas Hidrográficas (ENGICH) de Chile (CONAMA, 2007). Asimismo, es necesario incorporar estos antecedentes a consulta de expertos y a elementos de ingeniería hidráulica, para que sirvan como herramienta de apoyo en los procesos de toma de decisiones.

\section{CONCLUSIÓN}

El modelo hidrológico creado con ArcHydro en plataforma SIG y aplicado a las cuencas de los ríos Moncul y Budi proporciona antecedentes básicos para caracterizar y comparar las cuencas, logrando un modelo del funcionamiento, regionalización y representación de la red hídrica a escala 1:50.000.

La aplicación de parámetros de medición rápida al modelo hidrológico, permiten inferir que la cuenca del Budi tiene una renovación hídrica menor y es más susceptible a actividades antrópicas y naturales que Moncul. Esto, fundado en su perfil latitudinal menor, la forma de la red de drenaje centrípeto endo-exorreica y el tipo de uso del suelo que lo deja descubierto gran parte del año.

Los resultados representan información de base para la toma de decisiones sobre el manejo de recursos hídricos, sobre todo cuando existe carencia de datos de terreno.

\section{BibLIOGRAFíA}

Allewijn, R. (1988): "Regional hydrological system analysis using remote sensing data and geographical information system, Application to groundwater modeling of the Roermod area, The Netherlands", International Journal of Remote Sensing, 9/10, pp. 1775-1785. 
Al-Sabhan, W., Mulligan, M. y Blackburn, G. (2003): “A real-time hydrological model for flood prediction using GIS and the WWW", Computers, Environment and Urban System, 27/1, pp. 9-32.

Avila, M. (2002): "Modelos digitais de elevação hidrologicamente consistentes para a bacia amazônica", tesis doctoral inédita, Universidade Federal de Viçosa, Minas Gerais, Brasil, 132 pp. http://www.bibliotecaflorestal.ufv.br/bitstream/handle/ 123456789/322/108161_c.pdf?sequence=2 (Fecha de consulta: 07/04/2016).

Belmonte, S. y Nuñez, V. (2006): "Desarrollo de modelos hidrológicos con herramientas SIG", Revista Internacional de Ciencia y Tecnología de la Información Geográfica, Geofocus, 6, pp. 15-27.

Boyko, O. y Treebushny, D. (2006): "Application of a physically based distributed rainfall-runoff model TOPKAPI-IMMSP to gauged and ungauged mountainous catchments of ranscarpathean region, Ukraine", Geophysical Research Abstracts, 17/5, pp. 637-644.

Campos, D. (1998): Procesos del ciclo hidrológico, México, Editorial Universitaria Potosiana.

Centro de Estudios de Ordenamiento del Territorio y Medio Ambiente (CEOTMA) (1981): Guía para la elaboración de estudios del medio físico: contenido y metodología, Madrid, MOP, 809 pp.

Choi, W. y Deal, B. (2008): "Assessing hydrological impacto of potencial land use change through hydrological and land use change modeling for the Kishwaukee River Basing (USA)", Journal of Environmental Management, 88/4, pp. 1119-1130.

Chuvieco, E. (2008): Teledetección ambiental, La observación de la Tierra desde el Espacio, Barcelona, Ariel, 594 pp.

Comisión Nacional del Medio Ambiente (CONAMA) (2007): Estrategia Nacional de Gestión Integrada de Cuencas Hidrograficas, Santiago de Chile, Gobierno de Chile, $57 \mathrm{pp}$.

Conde, C. y Baena J. (2004): "SIG y Paleohidrología: Reconstrucción del modelo hidrológico en el yacimiento paleolítico del Camino de Salmedina", CuPAUAM: Cuadernos de Prehistoria y Arqueología, 30, pp. 9-25.

Corporación Nacional Forestal - Comisión Nacional del Medio Ambiente (CONAF-CONAMA) (1999): Catastro y evaluación de recursos vegetacionales nativos de Chile, Informe regional IX Región, Proyecto CONAF-CONAMA-BIRF, Santiago de Chile, 90 pp.

Corporación Nacional Forestal - Comisión Nacional del Medio Ambiente (CONAFCONAMA) (2007): Actualización del catastro y evaluación de recursos vegetacionales nativos de Chile, Informe regional IX Región, Proyecto CONAF-CONAMA-BIRF, Santiago de Chile, 90 pp.

Di Castri, F. y Hajek, E. (1975): Bioclimatografía de Chile, Santiago de Chile, Dirección de Investigación Vice-rectoría Académica, Universidad Católica de Chile, 170 pp.

Doffo, N. y González Bonorino, G. (2005): "Caracterización morfométrica de la cuenca alta del arroyo Las Lajas, Córdoba: Un análisis estadístico", Revista de la Asociación Geológica Argentina, 60/1, pp. 16-22. 
Ferrando, J. (1994): "Métodos hidromorfométricos para determinar la erosividad en cuencas hidrográficas", Ingeniería Hidráulica en México, IX/3, pp. 5-14.

García, S., Francés, F. y Andreu, J. (2002): "Simulación hidrológica basada en SIG: Sensibilidad a factores de escala”, Ingeniería del Agua, 9/3, pp. 295-308.

Gopalan, H. (2003): "WRAP Hydro Data Model: Finding Input Parameters for the Water Rights Analysis Package", Master of Science in Engineering, Austin, The University of Texas.

Henao, S. (1988): Introducción al manejo de cuencas hidrográficas, Universidad Santo Tomás, Centro de enseñanza desescolarizada, Bogotá, Ediciones USTA, 396 pp.

Hesse, C., Krysanova, V., Päzolt, J. y Hattermann, F. (2008): "Eco-hydrological modeling in a highly regulated lowland catchment to find measures for improving water quality", Ecological Modelling, 218, pp. 135-148.

Himmelblau, D. y Bischoff, K. (2004): Análisis y Simulación de Procesos, Barcelona, Editorial Reverté S.A.

Immerzeel, W. y Droogers, P. (2008): "Calibration of a distributed hydrological model based on satellite evapotranspiration", Journal of Hydrology, 349, pp. 411-424.

Instituto Nacional de Estadísticas (INE) (2002): "Censo de población y vivienda nacional año 2002", http://www.ine.cl/canales/usuarios/censos_digitalizados.php (Fecha de consulta: 07/04/2016).

Jardí, M. (1985): "Forma de una cuenca de drenaje. Análisis de las variables morfométricas que nos la definen", Revista de Geografía, 19, pp. 41-68.

Llorens, P., Oliveiras, I. y Poyatos, R. (2003): "Temporal variability of water fluxes in a Pinus sylvestris Forest patch in Mediterranean mountain conditions", en E. Servat, W. Najem, C. Leduc, y A. Shakeel (eds.), Hydrology of Mediterranean and semiarid regions, United Kindom, IAHS publication 278, pp.101-105.

Llorente, M., Díez-Herrero, A. y Laín, L. (2009): "Aplicaciones de los SIG al análisis y gestión del riesgo de inundaciones: avances recientes", Cuadernos de la Sociedad Española de Ciencias Forestales, 29, pp. 29-37.

Loaiza, J. (2007): "Soil hydrology in the Ribera Salada Catchment (Catalan Pre Pyrenees) Application of hydrologic models for the estimation of hydrologic transitional regimes", Doctor (PhD) in Soil sciences and environment, Universitat de Lleida.

Maidment, D. (2002): ArcHydro GIS for water resources, Redlands, ESRI Press, 224 pp.

Mark, D. (1983): "Relation between field-surveyed channel network and map-based geomorphometric measures, Inez Kentucky", Annals of the Association of American Geographers, 73/3, pp. 358-372.

Maza, J., Fernández, P., Vargas, A., Fornero, L., Trípodi, D., Yañez, H. y Nuñez, M. (1995): "Estudios hidrológicos e hidráulicos para el ordenamiento territorial de una Cuenca", Ingeniería del Agua, 2/1, pp. 31-44.

Mccoll, C. y Aggett, G. (2007): "Land-use forecasting and hydrologic model integration for improved land-use decision support", Journal or Environmental Management, 84, pp. 494-512. 
Mendoza, M., Bocco, G., Bravo, M., Siebe, C. y Ortiz, M. (2002): "Modelamiento hidrológico espacialmente distribuido: una revisión de sus componentes, niveles de integración e implicaciones en la estimación de procesos hidrológicos en cuencas no instrumentadas", Investigaciones Geográficas, 47, pp. 36-58.

Miller, S., Semmens, D., Miller, R., Goodrich, D., Hernandez, M., Miller, R., Kepner, W. y Phillipe, W. (2007): "The automated geospatial watershed assessment tool", Environmental Modelling \& Software, 2/3, pp. 365-377.

Miller, R., Guertin, D. y Heilman, P. (2004): "Information technology in watershed management decision making", Journal of the American Water Resources Association, 40/2, pp. 349-357.

Moreno, J. y Salazar, J. (2008): "Generación de series sintéticas de caudales usando un Modelo Matalas con medias condicionadas", Avances en Recursos Hidráulicos, 17, pp. 17-24.

Moreno, J. y Salazar, J. (2009): "Modelo autoregresivo multivariado basado en regímenes para la Generación de Series Hidrológicas", Dyna, 76/157, pp. 101-108.

Moussa, R., Chaihinian, N. y Bocquillon, C. (2007): "Distributed hydrological modelling of a Mediterranean mountainous catchment - Model construction and multisite validation", Journal of Hydrology, 337, pp. 35-51.

Müller-Wohlfeil, D., Lahmer, W., Krysanova, V. y Becker, A. (1996): Topography-based hydrological modelling in the Elbe drainage basin, Proceedings Third International Conference/Wokshop on Integrating SIG and Environmental Modeling, Santa Fe, NM.

Núñez, F., Castro, F., Saraiva, M. y Ramos, I. (1998): "Coupling GIS with Hydrologic and Hydraulic Flood Modelling", Water Resources Management, 12/3, pp. 229-249.

O Callaghan, J. y Mark, D. (1984): "The extraction of drainage networks from digital elevation data", Computer Visions, Graphics and Image Processing, 28/3, pp. 323-44.

Olaya, V. (2004): "Hidrología Computacional y Modelos Digitales de Terreno, Teoría, práctica y filosofía de una nueva forma de análisis hidrológico", http://www.gabrielortiz.com/descargas/Hidrologia_Computacional_MDT_SIG.pdf (Fecha de consulta: 07/04/2016).

Olivares, A. (2004): "Modelización hidrológica Pseudo-Distribuída del Barranc del Carraixet: Aplicación al episodio de octubre de 2000", Cuadernos de Geografía, 76, pp. 155-182.

Ozdemir, H. y Bird, D. (2009): "Evaluation of morphometric parameters of drainage Networks derived from topographic maps and DEM in point of floods", Environmental Geology, 56/7, pp. 1405-1415.

Palacios, E. (2004): "Modelo para manejo de la incertidumbre hidrológica en la planificación de la operación del Sistema Interconectado Central (SIC)", tesis de ingeniería civil eléctrica, Universidad de Chile, Santiago de Chile, http://www.centro energia.cl/literatura/memorias_tesis/MemoriaEugenioPalacios.pdf (Fecha de consulta: 07/04/2016).

Patton, D. (1975): "A Diversity index for quantifying habitat edge", Wildlife Society Bulletin, 3, pp. 171-173. 
Pedregal, B. (2005): Población y planificación hidrológica, Sevilla, Universidad de Sevilla y Junta de Andalucía, 408 pp.

Peña-Cortés, F., Escalona, M., Pincheira-Ulbrich, J. y Rebolledo, G. (2011): “Cambio de uso del suelo en los geosistemas de la cuenca costera del Río Boroa (Chile) entre 1994 y 2004", Revista de la Facultad de Ciencias Agrarias Universidad de Cuyo, 43/2, pp. 1-20.

Peña-Cortés, F., Escalona-Ulloa, M., Rebolledo, G., Pincheira-Ulbrich, J. y Torres-Álvarez, O. (2009): "Efecto del cambio en el uso del suelo en la economía local: Una perspectiva histórica en el borde costero de la Araucanía, sur de Chile", en U. Confalonieri, M. Mendoza y L. Fernández (eds.), Efecto de los cambios globales sobre la salud humana y seguridad alimentaria, Argentina, Red CYTED 406RT0285, pp. 184-197.

Peña-Cortés, F., Gutiérrez, P., Rebolledo, G., Escalona, M., Hauenstein, E., Bertrán, C., Schlatter, R. y Tapia, J. (2006): "Determinación del nivel de antropización de humedales como criterio para la planificación ecológica de la cuenca del lago Budi, IX Región de La Araucanía, Chile", Revista de Geografía Norte Grande, 36, pp. 75-91.

Peña-Cortés, F., Limpert, C., Andrade, E., Hauenstein, E., Tapia, J., Bertrán, C. y Vargas-Chacoff L. (2014): "Dinámica geomorfológica de la costa de La Araucanía", Revista de Geografía Norte Grande, 58, pp. 241-260.

Peña-Cortés, F., Pincheira-Ulbrich, J., Bertrán, C., Tapia, J., Hauenstein, E., Fernández, E. y Rozas, D. (2011): "A study of the geographic distribution of swamp forest in the coastal zone of the Araucanía Region, Chile", Applied Geography 31/2, pp. 545-55.

Pincheira-Ulbrich, J., Rau, J. y Peña-Cortés, F. (2009): "Tamaño y forma de fragmentos de bosque y su relación con la riqueza de especies de árboles y arbustos", Phyton, 78, pp. 121-128.

Pizarro, R., Soto, M., Farias, C. y Jordan, C. (2005): "Aplicación de dos Modelos de Simulación Integral Hidrológica, para la estimación de caudales medios mensuales, en dos cuencas de Chile central", Bosque, 26/2, pp. 123-129.

Pusineri, G., Pedraza, R. y Lozeco, C. (2005): "Uso de modelos digitales de elevación y de sistemas de información geográfica en la modelación hidrológica", Primera Reunión de Usuarios de Sistemas de Información Geográfica y Procesadores Digitales, Universidad Nacional del Nordeste, Argentina. http://hum.unne.edu.ar/revistas/geoweb/Geo4/archivos/pusineri.pdf (Fecha de consulta: 07/04/2016).

Rovira, A. y Latorre, P. (1986): "Percepción del riesgo de inundaciones en la comuna de San Bernardo", Ambiente y Desarrollo, 2/3, pp. 123-129.

Salazar, C. (2003): "Situación de los recursos hídricos en Chile, Centro del Tercer Mundo para el Manejo del Agua", México. http://www.thirdworldcentre.org/ publi.html (Fecha de consulta: 07/04/2016).

Sharma, S. y Anjaneyulu, D. (1993): "Application of remote sensing and GIS in water resource management", International Journal of Remote Sensing, 14/17, pp. 32093220 . 
Sharma, S. y Singh, S. (1995): "Satellite remote sensing for soil erosion modeling the ANSWER model”, International Journal of Remote Sensing, 13/9, pp. 259-272. Smart, J. (1972): "Channel networks", Advances in Hydroscience, 8, pp. 305-346. Strahler, A. y H Strahler, A. (1989): Geografía física, Barcelona, Omega, 636 pp. Tarboton, D., Bras, R. y Rodriguez-Iturbe, I. (1991): "On the extraction of channel networks from digital elevation data", Hydrological Processes, 5, pp. 81-100.

Thunnissen, H., Jaarma, M. y Schoumans, O. (1992): "Land cover inventory in the Netherlands using remote sensing, application in a soil and water vulnerability assessment system", International Journal or Remote Sensing, 13/9, pp. 1693-1708.

Utton, A. (1985): "In search of an integrating principle for interstate water law: regulation versus the market place", Natural Resources Journal, 25, pp. 984-1004.

Velásquez, J., Rivera, J. y Suárez, M. (2007): Implementación del modelo de datos ArcHydro en la región hidrológica Balsas, México, Instituto Mexicano de Tecnología del Agua (IMTA). http://www.inecc.gob.mx/descargas/cuencas/cong_nal_06/tema_ 05/13_jaime_velazquez.pdf (Fecha de consulta: 07/04/2016).

Viviroli, D., Zappa, M. y Weingartner, R. (2009): "An introduction to the hydrological modelling system PREVAH and its pre- and post- processing-tools", Environmental Modelling E Software, 24, pp. 1209-1222.

Fecha de recepción: 24 de octubre de 2014.

Fecha de aceptación: 14 de julio de 2015.

\section{RESUMEN}

Los modelos hidrológicos espacialmente distribuidos han sido una potente herramienta para el levantamiento indirecto de información de una cuenca hidrográfica. En este estudio, se caracteriza el comportamiento hidrológico de dos cuencas localizadas en el borde costero de la región de La Araucanía. El método se basó en un proceso de modelación hidrológica espacialmente distribuida con los softwares ArcHydro y WARPHydro. Los resultados mostraron diferencias en el comportamiento hidrológico entre ambas cuencas atribuidas a la forma de la red hídrica, altitud y escorrentía superficial. Se concluye que cuando existe carencia de datos de terreno y los costos limitan el uso de otros métodos, la modelación hidrológica genera información de base para la toma de decisiones para el manejo del agua.

Palabras Clave: modelación hidrológica; gestión de recursos hídricos; herramienta ArcHydro; herramienta WarpHydro; red de drenaje.

\section{AbSTRACT}

Spatially distributed hydrological models have been a powerful tool for indirect survey information of a watershed. In this study, the hydrological behavior of two basins located on the coastal edge of the Araucanía region is characterized. The method is based on a process of spatially distributed hydrological modeling with WARPHydro 
and ArcHydro softwares. The results showed differences in behavior between the two hydrological basins attributed to the shape of the water supply, altitude and surface runoff. We conclude that when there is a lack of field data and costs limit the use of other methods, hydrologic modeling generates information base for decision-making on water management.

KeY wORDS: Hydrologic Modeling; Water Resources Management; Biophysical characterization; ArcHydro Tools; WarpHidro Tools; Drainage net. 